\title{
Gene expression live on view
}

Expression of c-fos mRNA and protein in brain slices has been used as a marker of neuronal activity. Liu et al. describe a new technique in which c-fos mRNA is magnetically labelled, allowing in vivo visualization of this marker in mice by MRI.

The researchers created singlestranded oligonucleotide sequences that were complementary to those of c-fos mRNA and labelled them with fluorescein isothiocyanate (FITC), so that they could be visualized in histological sections. These oligonucleotide sequences were linked to supramagnetic iron oxide nanoparticles (SPIONs), creating SPION-c-fos probes that could be used as a contrast agent for MRI.

Liu and colleagues administered the SPION-c-fos probes to anaesthetized mice by intracerebroventricular infusion. MRI images of the mouse brains revealed SPION-c-fos retention not only in the tissue surrounding the infusion site, but also in the contralateral cortex. This remained visible for at least 1 day, whereas there was no retention of SPIONs that were not linked to the oligonucleotide sequence. Histological sections of the brains subsequently showed that the FITC-labelled oligonucleotides colocalized with iron oxide (stained using Prussian Blue), indicating that the two halves of the probe had remained linked in the brain. Further examination of the brain sections showed that the probe had been

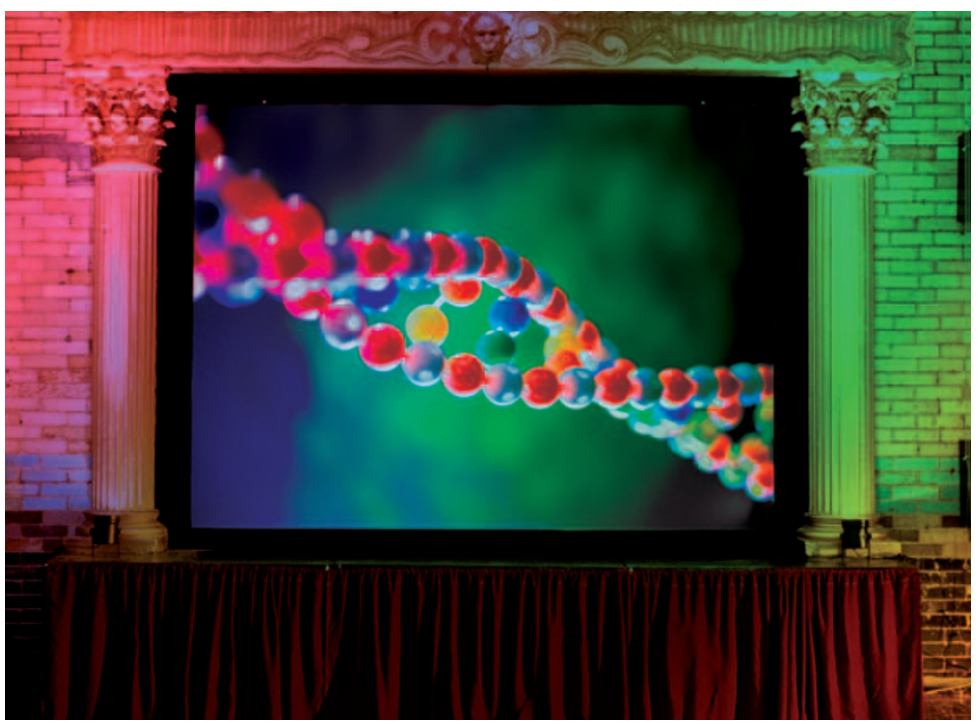

taken up by neurons but not by glial cells. Iron oxide was present in the hippocampus, cerebellum and the ventricular wall, demonstrating that the probe had travelled up to $6 \mathrm{~mm}$ from the injection site. Importantly, the MRI signal correlated with c-fos mRNA expression patterns obtained by in situ hybridization.

In a separate experiment, the researchers injected mice with SPION-c-fos and 4 hours later with amphetamine, which is known to induce c-fos mRNA expression in the striatum and the forebrain. MRI scans taken three hours after the amphetamine injection showed a robustly increased SPION-c-fos retention in the nucleus accumbens, striatum and medial prefrontal cortex, showing that the visualized c-fos expression induced by amphetamine administration was consistent with patterns that had been detected using traditional methods.

This exciting new technique offers promising possibilities for assessing the effects of pharmacological and other manipulations on gene transcription in the brains of live animals and, when non-invasive methods of probe administration have been developed, in human brains.

Leonie Welberg

ORIGINAL RESEARCH PAPER Liu, C. H. et al.

Imaging cerebral gene transcripts in live animals. J. Neurosci. 27, 713-722 (2007) 